\title{
Physical Typologies of Older Adults Living in a Retirement Village
}

ISSN: 2578-0093

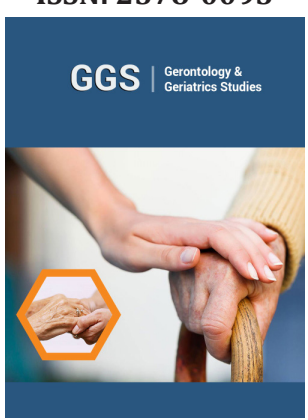

*Corresponding author: Carol Wham, School of Sport, Exercise and Nutrition, Massey University, New Zealand

Submission: 㭗 October 18, 2019

Published: 眥October 31, 2019

Volume 5 - Issue 3

How to cite this article: De Villiers B, Ali A, Wham C. Physical Typologies of Older Adults Living in a Retirement Village. Gerontol \& Geriatric stud.5(3). GGS.000611.2019.

DOI: 10.31031/GGS.2019.05.000611

Copyright@ Carol Wham, This article is distributed under the terms of the Creative Commons Attribution 4.0 International License, which permits unrestricted use and redistribution provided that the original author and source are credited.

\author{
Baron De Villiers ${ }^{1}$, Ajmol Ali ${ }^{1,2}$ and Carol Wham ${ }^{1,2 *}$ \\ ${ }^{1}$ School of Sport, Exercise and Nutrition, Massey University, New Zealand \\ ${ }^{2}$ Centre for Metabolic Health Research, Massey University, New Zealand
}

\section{Abstract}

Retirement village accommodation is in demand and an opportune setting for healthy eating and physical activity. In this study 43 older men and women completed a nutrition knowledge questionnaire and physical measures; BMI, muscle mass, fat mass, grip strength, five times sit to stand, gait speed as well as physical activity and 7-day step count measured by accelerometers. Hierarchical clustering was used to rank participants according to their age and physical measures. Four distinct physical typologies emerged: 'strong sedentary', 'weak sedentary', 'overweight active' and 'lean active'. Strong sedentary and overweight active typologies were mostly men, stronger $(\mathrm{p}<0.05)$ with a higher BMI $(\mathrm{p}<0.001)$. The lean active group were mainly women, recorded the highest step counts $(\mathrm{p}<0.001)$, levels of activity $(\mathrm{p}<0.001)$ and were less sedentary $(p<0.001)$. High levels of nutrition knowledge $(>74 \%)$ were observed across all groups. Tailoring interventions to suit physical typologies may help modify eating and physical activity behaviors.

Keywords: Nutrition; Older adults; BMI; Physical function; Cluster analysis; Community

\section{Introduction}

Lifestyle factors such as diet and physical activity are key to maintaining good physical function and quality of life in older adults. Health problems are often associated with changes in body composition [1]. exacerbated by low levels of physical activity, sedentary lifestyles and poor nutrition habits. Adults over 65 years are the least physically active population group [2]. However, although regular physical activity and a healthy diet may help prevent non-communicable disease and disability $[3,4]$, older adults may be challenged by a lack of motivation. Retirement villages may provide a viable option to combat this problem with purpose-built housing and facilities for older adults to socialize and engage in physical activities. Villages may include a gymnasium or fitness studio, swimming pool, bowling greens and recreation rooms and offer social walking groups which can motivate residents to be more active and help prevent loneliness [5,6]. Nevertheless, although retirement villages offer a range of activities to promote physical activity these can have low attendance rates [7].

A successful six-month physical activity and nutrition intervention in retirement villages in Australia found older adults improved their weight, engagement in strength exercises, increased levels of physical activity and significantly increased frequency of fruit intake, along with fiber intake and fat avoidance [4]. An important component was the key role retirement village managers played as 'gate keepers' especially with their support for recruitment of residents to attend information sessions. This strategy led to a positive impact on dietary behaviors. Residents gained increased knowledge of appropriate food types and meals through the provision of educational sessions. Retirement is a major life change that provides an opportunity for older adults to modify their eating and activity behaviors. Residents in retirement villages appear to have sedentary lifestyles [4,8] and tailored physical activity and nutrition programs for older adults in retirement villages may help to counter functional decline. Presently the physical characteristics and level of nutrition knowledge among residents in retirement villages is unknown. Considering the steady increase in the ageing population and demand for retirement village accommodation, understanding the need for positive health behavior changes is an important first step. This study aimed to characterize the physical typologies of older adults residing in a retirement village based on their age, anthropometry and levels of physical activity and to investigate their levels of nutrition knowledge. 


\section{Methods}

\section{Study population}

Residents aged $\geq 65$ years, living independently in a retirement village, who were willing to undertake physical measures (weight, height, body mass index (BMI), bioelectrical impedance analysis (BIA), grip strength, a gait speed test, five times sit-to-stand (FTSS) test) were invited to participate in this study through convenience sampling via poster boards, weekly newsletters and word of mouth throughout the village. Participants were excluded if they had an implantable cardioverter defibrillator (ICD) or pacemaker, were receiving any medical care that may affect food intake or physical activity and if they required career support. Ethical approval was provided by Massey University Human Ethics Committee (Southern A, SOA 18/09).

\section{Measures}

Anthropometry: Physical assessments included measures of height and weight and were conducted in the health clinic of the retirement village using standardized procedures. Skeletal muscle mass (SMM), body fat mass (BFM), and body fat percentage (BF\%) were estimated using bioimpedance (In Body 270, Seoul, South Korea).

Handgrip strength: Grip strength was measured using a handgrip dynamometer (Jamar hydraulic handgrip dynamometer, Patterson Medical, Bolingbrook, USA) using the dominant hand. Three measures were taken, with 60 -s recovery between bouts, and the maximum score was recorded as grip strength [9].

Five Times Sit to Stand (FTSS): The standard test protocol required participants to stand up and sit down as quickly as possible five times, keeping their arms folded across their chest [10]. A strong chair with seat height of $45 \mathrm{~cm}$ was used for each participant. The procedure was first demonstrated to the participant before they performed the first of two attempts. A 60-s rest was given in between each attempt. The faster of the two attempts was recorded.

Gait speed: Using a retractable tape measure, a 4-m line was marked. Following standard test protocol, participants were instructed to walk along a 4-m line from a stand still position with their feet behind a marked start line. Each participant's time was measured with a stopwatch after the first foot fall crossing the start line until the last foot fall at the finish line [11]. The test was performed twice and the faster of the two times was recorded.

Physical Activity: An accelerometer (GT3X, Actigraphy, Pensacola, USA) was provided to each participant with a waist strap. Participants were shown how to place it correctly on their dominant hip, either above or below their clothing. The accelerometer was set up to start recording at midnight on the given day and to stop recording after 7 days, using appropriate software (ACTi Life 6.7.0, Actigraphy, Pensacola, USA) [12]. Participants placed the accelerometers on their right or dominant hip upon waking or just after their shower or bath and accelerometers were left on throughout the course of the day for approximately 12 hours and only removed for swimming or showering. Accelerometers were removed before bed as sleep was not included as a measure. A 7-day step count, sedentary, light, moderate and vigorous physical activity intensity levels were recorded.

Nutrition Knowledge: Participants completed the Nutrition Attitude and Knowledge Questionnaire for Adults 50+ Years of Age (NAK-50+) [13], on a tablet (iPad, Apple, Cupertino, USA) using an online survey (SurveyMonkey, San Mateo, USA). The NAK-50+ is comprised of 27 items on general nutrition knowledge and attitudes (e.g., healthy weight, exercise, appetite, food groups, fluids as well as meal replacements and food enjoyment). Items are assessed using a 5-item Likert scale (totally agree, somewhat agree, somewhat disagree, totally disagree and don't know).

\section{Statistical analysis}

Statistical analyses were performed using IBM SPSS (v23 for Windows). Descriptive statistics were used to describe the participants' age, physical characteristics and nutrition knowledge scores. Hierarchical clustering was used to rank participants according to their age, physical measures (height, weight, BMI, skeletal muscle mass, body fat percentage, body fat mass), physical function test scores (handgrip strength, five times sit to stand, 4-m gait speed) and accelerometer measures (7-day step count, sedentary, light, moderate and vigorous physical activity intensity levels). This determined a hierarchical ranking of the participants' body composition measures and physical activity. The k-means clustering method was used to determine the final clusters. This method uses within-cluster variation for homogeneity and determined the number of clusters to be constructed (where $\mathrm{k}$ is equal to this number) and chooses a starting point for each of the clusters to be created by selecting an initial set of geometric centers called k centroids. The algorithm partitions the data into two or more clusters and performs an individual multiple regression on the data within each cluster [14]. Four clusters were determined based on the similarities/differences of anthropometric and physical activity variables between the clusters. Pearson's correlation coefficients were used to evaluate the dependence relationship between variables. $\mathrm{P}<0.05$ was considered statistically significant for all tests.

\section{Results}

A total of 20 men and 23 women (mean age 76 years), of New Zealand European ethnicity participated in the study. Participant characteristics by gender, including mean age, height, weight, BMI, $\mathrm{BFM}, \mathrm{BF} \%$, handgrip strength, FTSS, gait speed, accelerometer data and NAK-50+ scores are provided in Table 1 . The NAK-50+ mean scores were high (>74\% correct responses) and did not differ by gender. Accelerometer data showed women engaged in more light intensity activities $(\mathrm{p}=0.033)$ than men but no difference in steps, sedentary or other activity was observed. Highest response frequencies to each of the NAK-50+ questionnaire items are shown in Table 2. Responses to item 17 of the NAK-50+ "Water is the only type of fluid that is useful for hydration", were incorrect with "Somewhat agree" among cluster groups except the lean active group which responded correctly with "Somewhat disagree". Table 2 shows the participants' characteristics by four cluster groupings: 'strong sedentary', 'weak sedentary', 'overweight active' and 
'lean active' physical typologies. There was a main effect of BMI $(\mathrm{p}<0.001)$, with the strong sedentary and overweight active groups having a higher BMI than weak sedentary and lean active groups $(p<0.05)$. The strong sedentary and overweight active groups also had a higher grip strength $(\mathrm{p}<0.05)$ than the weak sedentary group. Times for FTSS did not differ between the groups $(p=0.355)$. Cluster group differences in four of the five accelerometer measures were evident. Those in the strong sedentary group took fewer steps than the overweight and lean active groups $(\mathrm{p}<0.001)$ and were more sedentary than other groups $(\mathrm{p}<0.001)$. Both the strong and weak sedentary groups undertook less light and moderate physical activity compared to the 'overweight active' and 'lean active' groups $(\mathrm{p}<0.001)$. There was a main effect of vigorous physical activity $(p=0.007)$, with the 'lean active group' engaging in higher intensity activity than the strong and weak sedentary groups $(\mathrm{p}<0.05)$.

Table 1: Mean (SD) Participant age, anthropometric and physical activity characteristics and NAK-50+ scores by gender.

\begin{tabular}{|c|c|c|c|c|}
\hline & $\operatorname{Men}(n=20)$ & Women $(n=23)$ & Total(n=43) & P-Value \\
\hline Age & $76.95(6.02)$ & $75.3(5.48)$ & $76.07(5.73)$ & 0.271 \\
\hline Weight (kg) & 81.771(14.09) & 65.03(11.01) & 72.81(14.99) & 0.001 \\
\hline BMI (kg/m2) & $27.19(3.52)$ & $25.27(4.2)$ & $26.17(3.97)$ & 0.296 \\
\hline BFM (kg) & $25.03(8.75)$ & $24.24(8.49)$ & $24.61(8.52)$ & 0.723 \\
\hline Body fat (\%) & 29.291(6.05) & $36.37(7.47)$ & $33.07(7.65)$ & 0.002 \\
\hline SMM (kg) & $32.22 \mathrm{a}(5.26)$ & $21.89(2.55)$ & $26.69(6.56)$ & $<0.001$ \\
\hline Handgrip (kg) & $32.911(8.05)$ & $18.43(4.11)$ & $25.16(9.56)$ & $<0.001$ \\
\hline FTSS (s) & $9.74(3.19)$ & $9.75(2.07)$ & $9.74(3.19)$ & 0.502 \\
\hline Gait Speed m/s) & $1.68(0.3)$ & $1.56(0.34)$ & $1.62(0.32)$ & 0.064 \\
\hline Step count & $47619(23812)$ & $48377(27515)$ & $48024(25558)$ & 0.709 \\
\hline Sedentary (\%) & $87.7(4.02)$ & $85.68(5.12)$ & $86.62(4.7)$ & 0.126 \\
\hline Light (\%) & $9.731(2.34)$ & $11.79(3.82)$ & $10.83(3.34)$ & 0.033 \\
\hline Moderate (\%) & $2.48(1.93)$ & $2.47(1.84)$ & $2.48(1.86)$ & 0.97 \\
\hline Vigorous (\%) & $0.09(0.31)$ & $0.06(0.11)$ & $0.07(0.22)$ & 0.662 \\
\hline NAK-50+ (\%) & 74.3(7.37) & 74.35(5.94)) & $74.33(6.56)$ & 0.372 \\
\hline
\end{tabular}

BMI: Body Mass Index; BFM: Body Fat Mass; SMM: Skeletal Muscle Mass.

Table 2: Participant age, anthropometric and physical activity characteristics by cluster groupings.

\begin{tabular}{|c|c|c|c|c|c|}
\hline n (\%) & $\begin{array}{c}1 \\
\text { Strong sedentary }(n=8) \\
(\text { Men=7; 87.5\%) }\end{array}$ & $\begin{array}{c}2 \\
\text { Weak sedentary }(n=16) \\
(M e n=4 ; 25 \%)\end{array}$ & $\begin{array}{c}3 \\
\text { Overweight active }(n=10) \\
\text { (Men=7; 70\%) }\end{array}$ & $\begin{array}{c}4 \\
\text { Lean active }(n=9) \\
(\text { Men=2; 22.2\%) }\end{array}$ & \\
\hline & Mean (SD) & Mean (SD) & Mean (SD) & Mean (SD) & $P$-value \\
\hline Age(y) & $77.25(4.89)$ & $76.5(6.34)$ & $78.3(6.04)$ & $71.78(2.54)$ & 0.066 \\
\hline Height $(\mathrm{cm})$ & $175.23^{\mathrm{a}, \mathrm{c}}(9.81)$ & 161.06(7.09) & $168.801(5.14)$ & $165.13(9.72)$ & 0.002 \\
\hline Weight (kg) & $90.16^{\mathrm{a}, \mathrm{c}}(3.76)$ & $64.76^{\mathrm{b}}(6.66)$ & 81.51(11.21) & $62.05(11.55)$ & $<0.001$ \\
\hline BMI (kg/m2) & $29.38^{\mathrm{a}, \mathrm{c}}(3.51)$ & $25.09^{\mathrm{b}}(3.24)$ & $28.6(3.54)$ & $22.55(1.86)$ & $<0.001$ \\
\hline Fat mass (kg) & $27.423(12.37)$ & $23.13(7.31)$ & $29.003(7.19)$ & $16.512(3.14)$ & 0.007 \\
\hline Body fat $\%$ & 29.95(11.67) & $35.333(8.69)$ & $35.313(5.25)$ & $27.13(5.75)$ & 0.066 \\
\hline Muscle mass (kg) & $34.66^{\mathrm{a}, \mathrm{b}, \mathrm{c}}(6.22)$ & $22.342(3.25)$ & $28.95(3.73)$ & $24.83(6.72)$ & $<0.001$ \\
\hline Hand grip (kg) & $29.251(9.69)$ & $20.56^{\mathrm{b}}(6.83)$ & $29(7.5)$ & $25.44(13.02)$ & 0.073 \\
\hline FTSS (s) & $10.6(2.31)$ & $9.86(2.06)$ & $9.26(2.11)$ & $8.72(2.7)$ & 0.355 \\
\hline Gait speed (m/s) & $1.62(0.27)$ & $1.46(0.29)$ & $1.69(0.31)$ & $1.79(0.36)$ & 0.07 \\
\hline
\end{tabular}




\begin{tabular}{|c|c|c|c|c|c|}
\hline Step Count & $24958^{\mathrm{b}, \mathrm{c}}(10434)$ & $34644^{\mathrm{b}, \mathrm{c}}(13122)$ & 63661(15792) & $74940(26833)$ & $<0.001$ \\
\hline Sedentary activity $\%$ & $91.40^{\mathrm{a}, \mathrm{b}, \mathrm{c}}(2.08)$ & $88.65^{b, c}(3.16)$ & $84.493(3.32)$ & $81.14(3.23)$ & $<0.001$ \\
\hline Light physical activity \% & $7.69^{b, c}(1.91)$ & $9.70^{\mathrm{b}, \mathrm{c}}(2.72)$ & $12.02(3.13)$ & $14.3(1.68)$ & $<0.001$ \\
\hline $\begin{array}{c}\text { Moderate physical } \\
\text { activity } \%\end{array}$ & $0.91^{\mathrm{b}, \mathrm{c}}(0.81)$ & $1.64^{b, c}(0.7)$ & $3.46(1.67)$ & $4.25(2.21)$ & $<0.001$ \\
\hline $\begin{array}{c}\text { Vigorous physical activ- } \\
\text { ity } \%\end{array}$ & $0.003(0.01)$ & $0.03(0.01)$ & $0.03(0.02)$ & $0.31(0.45)$ & 0.007 \\
\hline
\end{tabular}

Difference by ANOVA ${ }^{a}$ Weak sedentary group, ${ }^{b}$ Overweight active group, ${ }^{c}$ Lean active group $(\mathrm{P}<0.05)$. BMI: Body Mass Index; FTSS: Five Times Sit to Stand.

\section{Discussion}

Four physical typologies were evident 'strong sedentary', 'weak sedentary', 'overweight active' and 'lean active' among this group of retirement village residents based on anthropometric measures and levels of physical activity. More men than women predominated the 'strong sedentary' and 'overweight active' groups whereas 'weak sedentary' and 'lean active' groups were predominated by women. The 'strong sedentary' group recorded the highest mean BMI $\left(29.38 \mathrm{~kg} / \mathrm{m}^{2}\right)$ and lower BF\% compared to the 'overweight active' and 'weak sedentary' and had a higher muscle mass than other groups $(\mathrm{p}<0.001)$. Compared to their predominantly male 'overweight active' counterparts mean grip strength did not differ, however the 'strong sedentary' group recorded significantly lower 7-day step counts, higher sedentary activity and lower levels of light and moderate physical activity. Muscle mass and strength are vitally important when ageing and may help prolong independence, health and inevitably a higher quality of life $[15,16]$ but the observation that higher muscle mass did not translate into greater muscle strength may suggest that higher levels of physical activity may benefit the 'strong sedentary'.

The 'lean active' group were mostly women, tended to be younger and had the lowest BMI and BF\% of all the groups. While grip strength of the 'lean active' group did not differ from the predominantly male 'strong sedentary' and 'overweight active' groups, they outperformed other groupings in gait speed, step count and all levels of physical activity. The physical activity levels observed in the 'lean active' group is encouraging but a possible concern is that mean $\mathrm{BMI}<23$ is at the lower end of the recommended BMI range. Evidence from a meta-analysis suggests a protective effect of a BMI $\geq 23$ [17] as there is an increased risk of mortality for older adults when $\mathrm{BMI}<23$. Further, positive associations between increasing BMI and improved functional status [18] suggests the 'lean active' may need regular weight checks for a healthy BMI to be maintained.

Overall, none of the participants fell below the cut-off thresholds for FTSS or gait speed which are effective assessments for loss of functional strength and mobility, respectively $[19,20]$. This may reflect the positive culture of the retirement village which provides a wide range of social activities, opportunities for physical activity and a dedicated gymnasium within the village. The physical activity findings observed warrant further investigation among other retirement establishments which are promoted as healthy lifestyle villages. Accelerometer data showed the 'overweight' and 'lean active' groups walked significantly more $(p<0.001)$ than the 'strong' and 'weak sedentary' groups. Normative step count data indicates healthy older adults undertake about 14,000 to 63,000 steps per week Tudor Locke et al. [21]. We observed the 'overweight' and 'lean active' groups averaged 63,661 and 74,940 steps per week, respectively, indicating above-average step-defined physical activity levels. The more sedentary groups were also above the normative ranges with the lowest step count observed in the 'strong sedentary 'group $(24,958)$ versus the 'weak sedentary' group which averaged 34,644 steps per week. We found higher step counts were observed in the predominantly female clusters compared to their male counterparts, however from a review of 28 prior studies step-defined physical activity of adults' $\geq 50$ years appears generally lower for women than men [21]. Generally, there are broad ranges of habitual activity evident from normative data which reflect the natural diversity of physical capacity common to older adults. Alongside well-known cardiovascular and metabolic health benefits, physically active older adults have higher levels of functional health, lower risks of falling, and improved cognitive health [22]. However, it appears there is no evidence to inform an absolutely defined moderate intensity cadence specific to older adults at this time [23]. Levels of nutrition knowledge among residents were high with both men and women scoring $>74 \%$ in the NAK-50+ questionnaire which suggests this small sample of older adults were actively engaged in their health and wellbeing. Responses to NAK-50+ questionnaire items differed across clusters only for the statement "Water is the only type of fluid that is useful for hydration" where the 'lean active' group only selected the correct response. Understanding fluid needs for hydration may be more relevant for this group who engage in higher levels of physical activity. Although we found nutrition knowledge was not related to physical measures or levels of physical activity, higher nutritionrelated knowledge has been found to be related to lower BMI and higher physical activity level among healthy European men and women aged 65 to 79 years [24]. This suggests that having a good nutrition-related knowledge and attitudes might positively impact on health and quality of life.

This study is limited by the small sample size of participants who identified as New Zealand European and lived in a high socioeconomic retirement village and is not representative of the older adult population. While we were able to pilot a wide range of body compositional and physical activity measures to generate 
different physical typologies the small convenience sampling limited the opportunity to engage older adults of different ethnicities and vulnerabilities. Nevertheless, the four physical typologies identified suggest tailored nutrition and physical activity interventions may together help to improve muscle mass, strength and physical function.

\section{References}

1. Baumgartner RN (2000) Body composition in healthy aging. Ann N Y Acad Sci 904(1): 437-448.

2. (2016) Health loss in New Zealand 1990-2013: A report from the New Zealand burden of diseases, injuries and risk factors study. Ministry of Health.

3. Rejeski WJ, Axtell R, Fielding R, Katula J, King AC (2013) Promoting physical activity for elders with compromised function: the lifestyle interventions and independence for elders (LIFE) study physical activity intervention. Clin Interv Aging 8: 1119-1131.

4. Jancey J, Holt AM, Lee A, Kerr D, Robinson S, et al. (2017) Effects of a physical activity and nutrition program in retirement villages: a cluster randomized controlled trial. Int J Behav Nutr Phys Act 14(1): 92.

5. Yeung P, Good G, Donoghue KO, Spence S, Blanka R (2017) What matters most to people in retirement villages and their transition to residential aged care. Aotearoa NZ Social Work 29(4): 84-96.

6. Holt A, Lee AH, Jancey J, Kerr D, Howat P (2016) Are retirement villages Promoting active aging? J Aging Phys Act 24(3): 407-411.

7. Nathan A, Wood L, Corti BG (2014) Examining correlates of self-reported and objectively measured physical activity among retirement village residents. Austr J Ageing 33(4): 250-256.

8. Mihalko SL, Wickley KL, Sharpe BL (2006) Promoting physical activity in independent living communities. Med Sci Sports Exerc 38(1): 112-115.

9. Jentoft AJC, Bahat G, Bauer J, Boirie Y, Bruyère O, et al. (2010) Sarcopenia: revised European consensus on definition and diagnosis. Age Ageing 48(1): 16-31

10. Bohannon RW (2006) Reference values for the timed up and Go test: a descriptive meta-analysis. J Geriatr Phys Ther 29(2): 64-68.

11. Goldberg A, Schepens S (2011) Measurement error and minimum detectable change in 4-meter gait speed in older adults. Aging Clin Exp Res 23(5-6): 406-412.

12. Aggio DA, Sartini C, Papacosta O, Lennon LT, Ash S, et al. (2016) Cross- sectional associations of objectively measured physical activity and sedentary time with sarcopenia and sarcopenic obesity in older men. Prev Med 91: 264-272.

13. Ducak K, Keller H (2015) Development and initial reliability testing of NAK-50+: A nutrition attitude and knowledge questionnaire for adults 50+ years of age. Can J Diet Pract Res 77(1): 3-8.

14. Späth H (2014) Mathematical algorithms for linear regression. ( $\left.1^{\text {st }} \mathrm{edn}\right)$, Academic Press, USA, p. 338.

15. Cheek J, Ballantyne A, Byers L, Quan J (2007) From retirement village to residential aged care: what older people and their families say. Health Soc Care Community 15(1): 8-17.

16. Rolland Y, Czerwinski S, Abellan VKG, Morley JE, Cesari M, et al. (2008) Sarcopenia: its assessment, etiology, pathogenesis, consequences and future perspectives. J Nutr Health Aging 12(7): 433-450.

17. Winter JE, MacInnis RJ, Wattanapenpaiboon N, Nowson CA (2014) BMI and all-cause mortality in older adults: a meta-analysis. Am J Clin Nutr 99(4): 875-890.

18. Bahat G, Tufan F, Saka B, Akin S, Ozkaya H, et al. (2012) Which body mass index (BMI) is better in the elderly for functional status? Arch Gerontol Geriatr 54(1): 78-81.

19. Cesari M, Kritchevsky SB, Penninx BW, Nicklas BJ, Simonsick EM, et al. (2005) Prognostic value of usual gait speed in well-functioning older people-results from the health, aging and body composition study. J Am Geriatr Soc 53(10): 1675-1680.

20. Goldberg A, Chavis M, Watkins J, Wilson T (2012) The five-times-sit-tostand test: validity, reliability and detectable change in older females. Aging Clin Exp Res 24(4): 339-344.

21. Locke CT, Hart TL (2009) Washington Expected values for pedometerdetermined physical activity in older populations.Int J Behav Nutr Phy Act 6(1): 59.

22. (2008) Physical Activity Guidelines Advisory Committee, Physical activity guidelines advisory committee report, 2008. DC: US Department of Health and Human Services, Washington, pp. A1-H14.

23. Locke CT, Craig CL, Aoyagi Y, Bell RC, Croteau KA, et al. (2011) How many steps/day are enough? For older adults and special populations. Int J Behav Nutr Phys Act 8(1): 80.

24. Bielak MJ, Dolowy AK, Santoro A, Ostan R, Berendsen AAM, et al. (2018) Are nutrition-related knowledge and attitudes reflected in lifestyle and health among elderly people? A Study Across Five European Countries. Front Physiol 9: 994. 